\section{Contrôle transcriptionnel des gènes ciliaires}

Jennifer Vieillard, Julie Jerber, Bénédicte Durand

$>$ Les cils, présents à la surface des cellules de nombreux eucaryotes, partagent une architecture commune qui peut se décliner en de nombreuses variations au sein d'une même espèce. La genèse des cils et la mise en place de leur diversité requièrent l'implication de gènes spécifiques. Ce contrôle est assuré par au moins deux classes de facteurs de transcription: les facteurs RFX (regulatory factor $X$ ), essentiels à l'assemblage de la plupart des cils, et les facteurs FOXJ1 (forkhead box J1), régulateurs clés de la croissance des cils mobiles. Ces facteurs ont des cibles distinctes et communes, et peuvent coopérer pour permettre la formation des cils. En collaborant avec d'autres facteurs de transcription spécifiques de différents types cellulaires, ils participent également à la spécialisation des cils. L'identification des gènes cibles des facteurs RFX et FOXJl est apparue comme une stratégie efficace pour identifier de nouveaux gènes ciliaires potentiellement impliqués dans les ciliopathies. <

\section{Cils : une diversité de structures et de propriétés}

Les cils et les flagelles sont des organites complexes qui forment des extensions à la surface de nom-

$(\rightarrow)$ Voir la Synthèse de C. Fort et P. Bastin, page 955 de ce numéro breux types cellulaires [48] $(\rightarrow)$. Leur structure est fortement conservée, des organismes unicellulaires, tels que l'algue verte Chlamydomonas, jusqu'à l'homme. Chez les animaux, ils sont impliqués dans de nombreuses fonctions et jouent un rôle important dans l'homéostasie des tissus, le développement et la reproduction [49] $(\rightarrow)$.

$(\rightarrow)$ Voir la synthèse de N. Diguet et S.M. Meilhac, page 996 de ce numéro
Cet article fait partie du numéro thématique de médecine/sciences intitulé «Cils primaires et ciliopathies $\left(\mathrm{m} / \mathrm{s} \mathrm{n}^{\circ} 11\right.$, vol. 30 , novembre 2014).

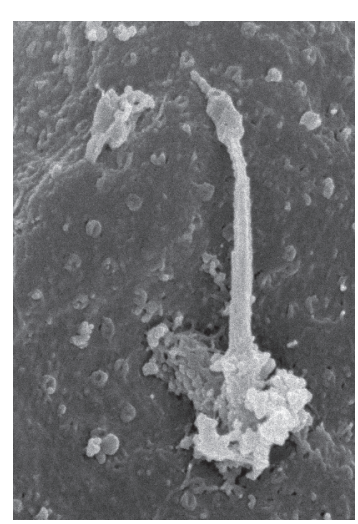

Centre de génétique et de physiologie moléculaire et cellulaire, UMR 5534 CNRS, université Claude Bernard Lyon-1, 16, rue Raphaël Dubois, 69622 Villeurbanne, France. benedicte.durand@univ-lyonl.fr ${ }^{1}$ J.V. et J.J. ont contribué de manière équivalente à l'écriture de cet article.

Les cils sont constitués de plusieurs centaines de protéines, certaines communes à tous les cils, d'autres spécifiques à un type de cil. Les programmes de transcription nécessaires à l'expression des gènes codant pour des protéines ciliaires commencent à être connus chez les animaux, mais ils ne sont cependant pas complètement élucidés [1]. Au niveau structural, les cils et les flagelles sont constitués d'une ossature microtubulaire, l'axonème, qui est nucléée à partir du corps basal, un $(\rightarrow)$ Voir la Synthèse de C. Fort et P. Bastin, page 955 de ce numéro dérivé du centriole père [48] $(\rightarrow)$.

Celui-ci est ancré à la membrane plasmique au niveau d'une structure particulière, la zone de transition, assemblée au-dessus du corps basal et qui joue un rôle dans le contrôle du passage des composants ciliaires dans le cil (Figure 1A). Le cil est entouré d'une membrane ciliaire en continuité avec la membrane plasmique, bien que sa composition en soit distincte. Il est isolé du reste de la cellule par la zone de transition et est assemblé grâce à un transport bidirectionnel, le transport intraflagellaire (IFT), qui permet l'acheminement de molécules dans et hors du cil [2, 3].

Différents types de cils sont décrits selon leurs structures ou leurs propriétés fonctionnelles. Les cils primaires, qui sont constitués de neuf doublets de microtubules périphériques $(9+0)$, sont présents en un seul exemplaire sur la plupart des cellules des mammifères au cours de la vie embryonnaire, et sur de nombreux tissus chez l'adulte, comme par exemple dans le rein où ils assurent une fonction mécanosensorielle (Figure IB). Ils jouent un rôle dans la perception de signaux extracellulaires, notamment dans la transduction de voies de signalisation, dont la voie Sonic Hedgehog (Shh) qui est essentielle au développement de nombreux organes [49] $(\rightarrow)$.

Par opposition, on distingue les cils qui possèdent,

$\rightarrow$ Voir la Synthèse de N. Diguet et S.M. Meilhac, page 996 de ce numéro en plus de neuf doublets périphériques, une paire centrale de microtubules $(9+2)$. La plupart de ces cils sont mobiles et possèdent différentes 


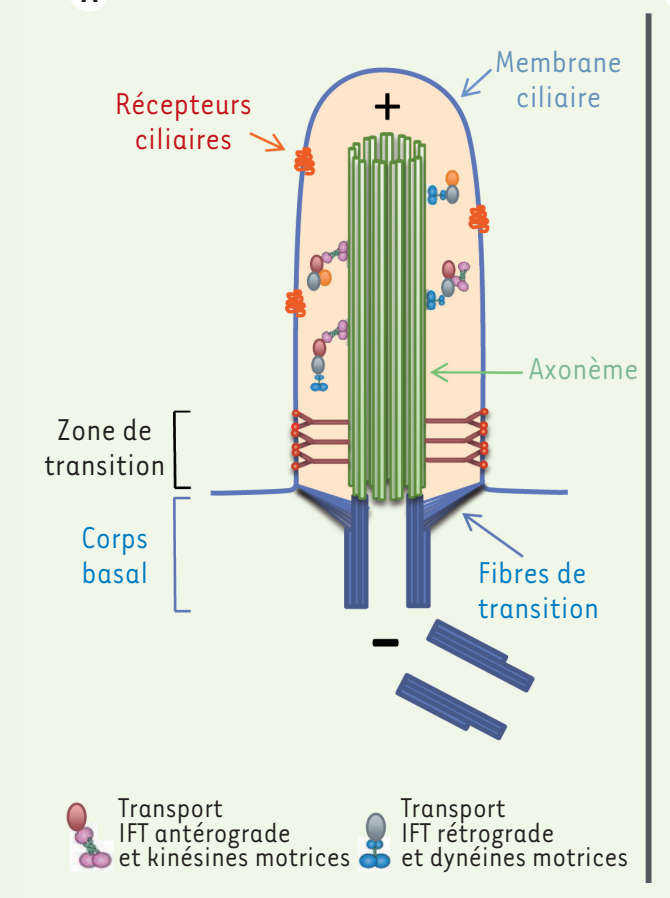

B
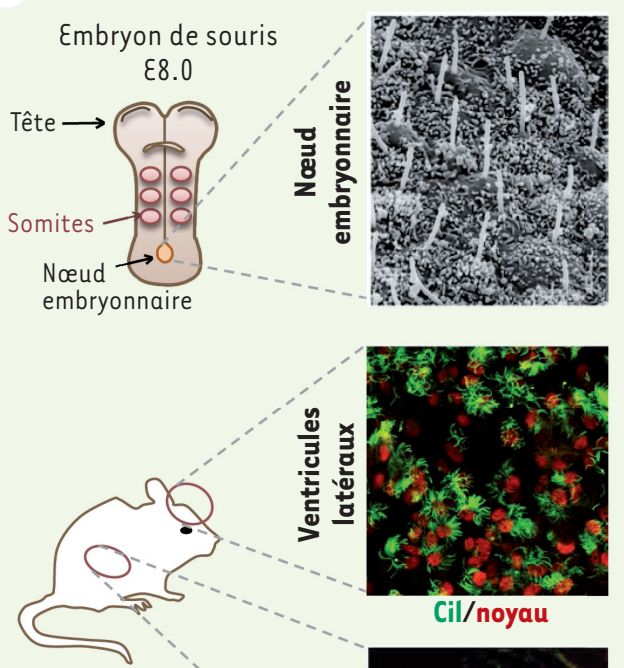

Ultrastructure du cil
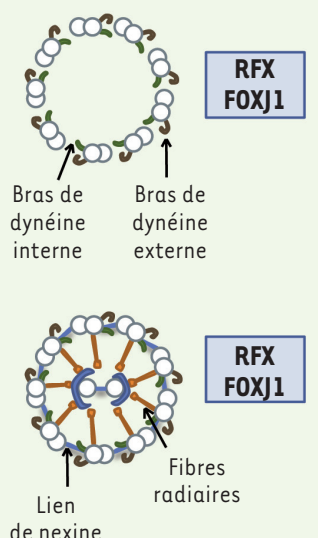

de nexine

ou N-DRC
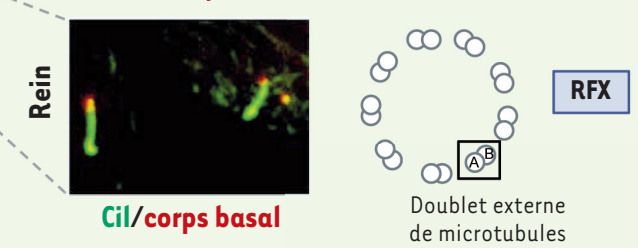

Figure 1. Représentation schématique d'un cil et exemples de la diversité des cils présents chez la souris. A. Le cil est nucléé à partir du corps basal qui s'ancre au niveau de la membrane plasmique via les fibres de transition. Le passage des protéines ciliaires à l'entrée et à la sortie du cil est régulé par une barrière sélective, la zone de transition. L'axonème du cil est allongé à partir du corps basal grâce à un mécanisme de transport bidirectionnel appelé transport intraflagellaire (IFT), qui fait intervenir des moteurs moléculaires chargés d'acheminer les constituants ciliaires depuis la base jusqu'à l'extrémité distale et vice-versa. $B$. Dans l'embryon de souris à $\varepsilon 8,0$, les cellules du nœud embryonnaire possèdent un cil primaire qui est mobile grâce à la présence de bras de dynéine internes et externes. Les cellules des ventricules latéraux possèdent de nombreux cils qui sont mobiles et qui permettent l'écoulement du liquide céphalorachidien. Leur axonème possède de nombreuses structures supplémentaires nécessaires au mouvement et aussi une paire centrale de microtubules. Au niveau des organes et en particulier du rein, les cellules possèdent un cil primaire non mobile qui assure des fonctions sensorielles essentielles pour le maintien de l'homéostasie cellulaire. Ce type de cil est aussi retrouvé sur les cellules de la majorité des organes et tissus de l'organisme au cours du développement embryonnaire et de la vie adulte. Deux familles de facteurs de transcription jouent un rôle clé dans l'assemblage des cils. De manière schématique, les facteurs de transcription RFX sont impliqués dans l'assemblage de la plupart des cils (mobiles ou non mobiles) en régulant l'expression de protéines requises pour la mise en place de tous les cils (protéines du transport intraflagellaire, BBSome, protéines de la zone de transition). Les facteurs de transcription FOXJl, quant à eux, sont principalement impliqués dans l'assemblage des cils mobiles en régulant des protéines nécessaires en particulier à la motilité ciliaire.

structures nécessaires à la motilité, telles que des bras de dynéine, des liens de nexine et des fibres radiaires (Figure 1B). Chez l'homme, ce type de cil est observé sous la forme de touffes de plusieurs cils par cellule au niveau des épithéliums respiratoires, des ventricules cérébraux et de l'oviducte. Le flagelle des spermatozoïdes, présent en un seul exemplaire sur chaque cellule, entre également dans cette catégorie de cils mobiles [3]. À côté de cette classification stéréotypée en deux catégories principales, il existe un ensemble de cils de structures variées. Par exemple, les cils des cellules du nœud embryonnaire (organe de latéralisation), qui sont essentiels à la mise en place de l'asymétrie droite/gauche au cours du développement des mammifères, possèdent une conformation en $(9+0)$ mais sont mobiles, grâce à la présence de bras de dynéine sur les doublets de microtubules périphériques (Figure 1B) $[4,49](\rightarrow)$.
À l'inverse, les neurones olfactifs des fosses nasales possèdent plusieurs cils de type $(9+2)$ par cellule, qui ne sont pas mobiles et ne possèdent pas de bras de dynéine [5]. De même, le kinocil, qui est nécessaire à l'organisation de l'épithélium auditif, est présent en une seule copie à la surface des cellules de l'oreille interne [50] $(\rightarrow)$. II possède une structure en

$(\rightarrow)$ Voir la Synthèse de J. Ezan et M. Montcouquiol, page 1004 de ce numéro $(9+2)$ avec des fibres radiaires et des bras de dynéine chez le poisson zèbre, mais pas de bras de dynéine chez la souris ; il ne semble malgré tout pas être mobile [6]. Enfin, dans les photorécepteurs, un cil en $(9+0)$ fait la jonction entre le segment interne et un segment externe qui, dans ce cas, est particulièrement développé et 
possède une morphologie unique adaptée à la fonction photoréceptrice. Ainsi, bien que leur structure de base soit commune, les cils peuvent varier considérablement d'un type cellulaire à l'autre en termes de taille, de forme, de nombre et de fonctions. De plus, ils sont assemblés selon un programme temporel bien précis qui diffère selon les cellules et les tissus. Cette diversité, qui est intimement liée aux différentes fonctions assurées par les cils, dépend de programmes transcriptionnels diversifiés, finement régulés et spécifiques du type cellulaire. Bien que ces programmes de transcription restent encore peu connus, deux grandes familles de facteurs de transcription sont plus particulièrement impliquées dans la ciliogenèse. Cependant, ces facteurs agissent souvent de concert avec d'autres facteurs spécifiques d'un type cellulaire pour augmenter la diversité ciliaire. Dans une première partie, nous aborderons les principaux facteurs de transcription régulant les gènes ciliaires, puis nous analyserons de quelle façon ils coopèrent pour participer à la diversité de structures et de fonctions des cils. Enfin, nous analyserons comment la recherche de nouveaux gènes cibles de ces facteurs de transcription peut permettre d'identifier de nouveaux gènes candidats des ciliopathies.

\section{Régulation transcriptionnelle des gènes ciliaires}

L'expression des gènes impliqués dans la ciliogenèse, c'est-à-dire des gènes codant pour des protéines nécessaires à la formation et à la fonction des cils, est principalement régulée par deux types de facteurs de transcription.

\section{Les protéines RFX}

Un premier groupe comporte les protéines RFX (regulatory factor $X$ ), membres de la famille des facteurs de transcription de type wingedhelix. Ces facteurs possèdent un domaine conservé de liaison à l'ADN et se fixent sur le promoteur de leurs gènes cibles au niveau d'une séquence consensus appelée boîte $X$. Ils ont été identifiés chez un grand nombre d'espèces eucaryotes. Huit facteurs RFX sont présents chez les mammifères, avec un facteur putatif supplémentaire chez le poisson zèbre. La drosophile possède deux facteurs RFX tandis que Caenorhabditis elegans et la levure n'en ont qu'un [7]. Ces facteurs peuvent être subdivisés en deux groupes selon qu'ils possèdent, ou non, un domaine de dimérisation. Ainsi, les protéines RFX1-4, RFX6 et RFX8 des mammifères, DAF-19 chez C. elegans et RFX-(CG6312) chez la drosophile possèdent ce domaine, alors que RFX5, RFX7 et le second RFX-(CG9727) de la drosophile n'en ont pas.

Parmi les facteurs possédant un domaine de dimérisation, DAF-19 chez C. elegans et RFX-(CG6312) chez la drosophile jouent un rôle essentiel dans la formation des cils des neurones sensoriels [8, 9]. Chez la souris, RFX3 est indispensable à l'élongation des cils du nœud embryonnaire [10] et des cellules des îlots de Langerhans du pancréas endocrine [11], ainsi qu'à la régulation du nombre, de la taille et de la motilité des cils dans les cellules épendymaires [12, 13]. D'autre part, les souris déficientes pour $R f x 4$ présentent des défauts de croissance des cils au niveau du système nerveux central [14]. Chez le poisson zèbre, Rfx2 est nécessaire à l'assemblage des cils de la vésicule de Kupffer, structure impliquée dans la mise

en place de l'asymétrie droite/gauche dans cet organisme [15]. Chez le xénope, Rfx2 est également nécessaire à la mise en place de l'épithélium mucociliaire [16]. II a été récemment démontré qu'il contrôle l'expression d'un grand nombre de gènes ciliaires [17]. La présence du domaine de dimérisation n'est pas systématiquement corrélée avec le contrôle des gènes ciliaires, puisqu'il a aussi été montré que le facteur $\operatorname{Rfx} 7$, qui ne possède pas ce domaine, est requis pour réguler la ciliogenèse dans le tube neural chez le xénope (Tableau I) [18]. De façon générale, les facteurs RFX régulent la ciliogenèse en contrôlant directement la transcription des gènes ciliaires et, notamment, de nombreux gènes codant pour des protéines essentielles à l'assemblage et à la fonction des cils [17, 19]. Ainsi, ils contrôlent l'expression de la majorité des protéines de la zone de transition nécessaires à l'ancrage du corps basal à la membrane plasmique, étape clé pour l'initiation de la ciliogenèse [51] $(\rightarrow)$. Ils contrôlent également l'expression des protéines du BBS (Bardet-Biedl syndrome)-ome, un complexe essentiel au transport des composants ciliaires et de la plupart des molécules de l'IFT (transport

$(\rightarrow)$ Voir la Synthèse de A. Benmerah, page 962 de ce numéro

$(\rightarrow)$ Voir la Synthèse
de $C$. Fort et $P$. Bastin,
page 955 de ce numéro intraflagellaire) [48] $(\rightarrow)$. Les facteurs RFX régulent aussi l'expression de gènes codant pour des protéines impliquées dans la motilité des cils, telles que certaines dynéines axonémales.

Certains facteurs RFX peuvent être coexprimés dans une même cellule. Ainsi, chez la souris, RFX3 et RFX4 sont tous deux exprimés dans certains territoires du cerveau, comme par exemple l'organe sous-commissural ${ }^{1}[13,20]$. RFX2 et RFX3 sont présents dans les cellules du nœud embryonnaire chez la souris $[10,15]$. Il en est de même pour les facteurs RFX1, RFX2 et RFX4, qui sont tous les trois exprimés dans les spermatides au cours de la spermatogenèse chez la souris [21]. Cette expression simultanée de plusieurs facteurs RFX capables de reconnaître un même motif d'ADN suggère une possible redondance fonctionnelle entre ceux-ci. Dans ce sens, il a été montré que deux facteurs RFX peuvent contrôler l'expression d'un même gène, comme c'est le cas pour RFX3 et RFX4, qui peuvent tous deux réguler l'expression du gène codant pour la protéine IFT172 [14]. Cependant, l'observation des phénotypes des souris déficientes pour les gènes $R f x 3$ ou $R f x 4$ suggère également que, dans certains territoires comme l'organe sous-commissural du cerveau, RFX3 et RFX4 coopèrent, car des défauts similaires sont observés dans ce territoire dans les deux modèles mutants $[13,20]$.

\footnotetext{
${ }^{1}$ Structure (d'origine épendymaire) qui borde le toit du $3^{e}$ ventricule du cerveau, spécialisée dans la sécrétion de glycoprotéines dans le liquide céphalorachidien.
} 


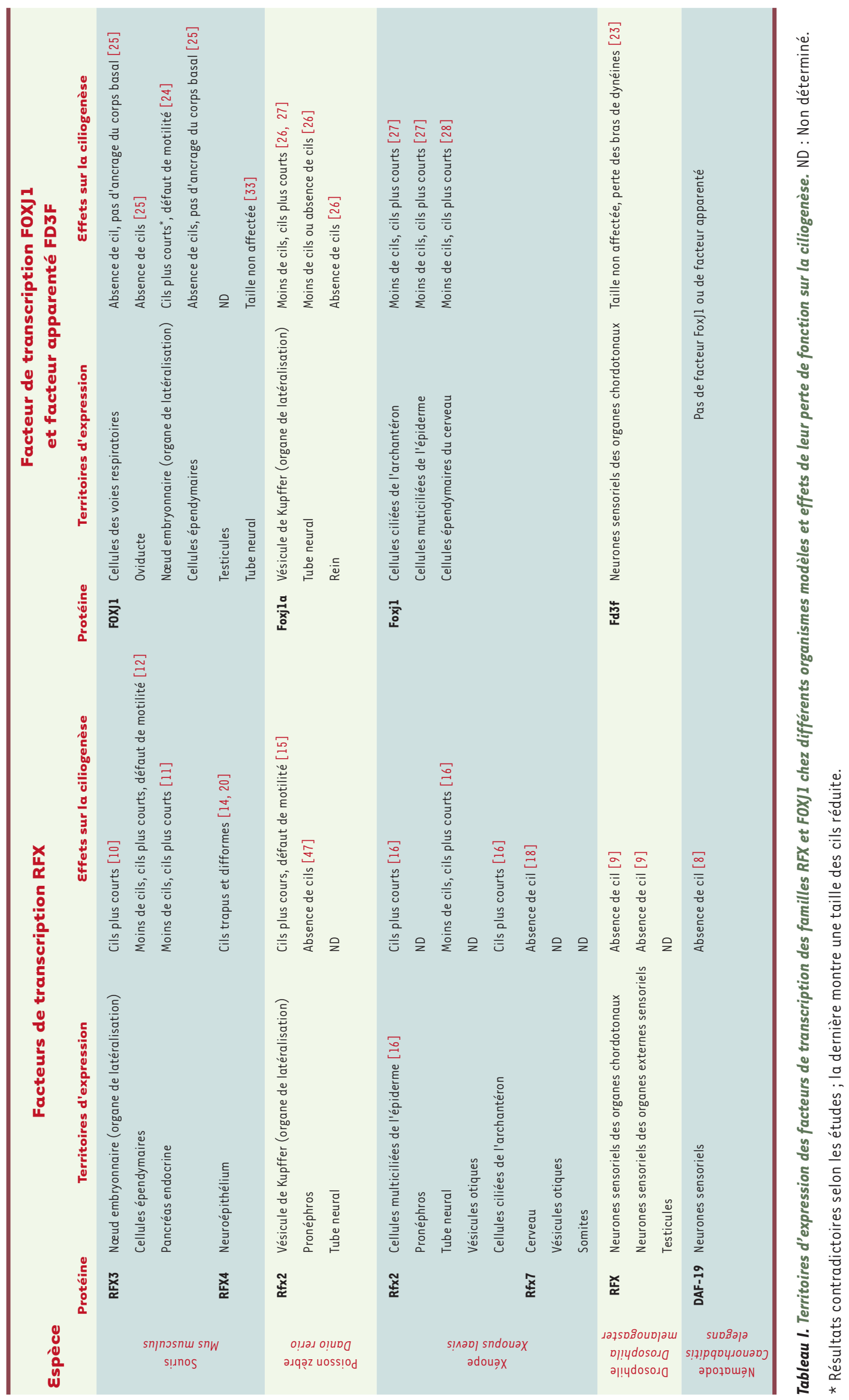

$\mathrm{m} / \mathrm{s} n^{\circ} 11$, vol. 30, novembre 2014 
De manière intéressante, les protéines RFX ne sont pas présentes chez les protozoaires ciliés et ne contrôlent donc pas la ciliogenèse de ces organismes. Leur recrutement au niveau des promoteurs des gènes ciliaires est apparu avec la multicellularité. Ceci est en faveur de I'hypothèse selon laquelle chez les mammifères, la ciliogenèse requiert la mise en place d'une régulation transcriptionnelle finement coordonnée au cours du temps et spécifique des tissus dans leurs différents territoires d'expression.

\section{Le facteur FOXJ 1}

Le second type de facteur de transcription requis pour la ciliogenèse est FOXJ1 (forkhead box J1), qui appartient à la famille des facteurs de transcription FOX, caractérisés par la présence d'un domaine particulier de liaison à I'ADN de type forkhead (FKH). Ce facteur est présent chez de nombreux eucaryotes et semble avoir évolué avec la présence de cils mobiles [22]. Aucun homologue n'est présent chez C. elegans qui ne possède pas de cils mobiles, ni chez Drosophila melanogaster. Cependant, chez la drosophile, le gène $f d 3 f$, apparenté à FOXJl, est nécessaire à la motilité des cils des neurones des organes chordotonaux $^{2}$ [23]. Chez les vertébrés, FOXJl est un acteur indispensable à l'établissement du programme de motilité des cils. Chez la souris, son absence induit des défauts de formation des cils mobiles dans de nombreux tissus, tels que le cerveau ou les épithéliums respiratoires. Cette altération est due à une défaillance de l'ancrage des corps basaux à la membrane plasmique. Deux études montrent également un défaut de formation et de motilité des cils du nœud embryonnaire $[24,25]$. Chez le poisson zèbre, la diminution d'expression de foxjla induit l'absence ou la réduction de la taille des cils mobiles présents dans la vésicule de Kupffer et le plancher du tube neural [26, 27]. Des défauts ciliaires similaires sont observés chez le xénope dans différentes régions lorsque l'expression de foxjl est diminuée (Tableau I) [28]. Plusieurs cibles de FoxJl ont été identifiées chez le xénope et le poisson, et sont impliquées, directement ou indirectement, dans la motilité des cils. Par exemple, Foxjl contrôle l'expression de gènes qui codent pour les protéines des bras de dynéine ou qui sont nécessaires à leur assemblage, comme LRRC6 (leucine rich repeat containing 6) qui, lorsqu'il est muté chez certains patients atteints de dyskinésie ciliaire primitive, affecte l'assemblage des dynéines axonémales dans les cils des voies respiratoires [29]. De manière remarquable, si FoxJl est nécessaire à l'assemblage des cils mobiles, il est également suffisant pour induire la formation de cils ectopiques dans des cellules épithéliales de poisson ou de xénope, ce qui justifie de considérer parfois ce gène comme un régulateur essentiel de la ciliogenèse [26, 27]. Toutefois, cette propriété ne semble pas être vraie dans toutes les cellules, ce qui montre également que le contrôle de la ciliogenèse ne peut se réduire à un seul facteur de transcription [30].

\footnotetext{
${ }^{2}$ Organes sensoriels internes des arthropodes qui répondent à l'étirement. Ils sont impliqués dans la proprioception et l'audition. On les retrouve entre autres en grand nombre au niveau de l'antenne de la drosophile, qui joue le rôle d'organe tympanique.
}

Coopération et régulation croisée des facteurs RFX et FOXJ1

En résumé, la régulation des gènes nécessaires à la ciliogenèse fait intervenir au moins deux grandes classes de facteurs de transcription dont chacune présente des spécificités d'action. Ceci nous permet déjà d'entrevoir comment la diversité des cils peut être engendrée. De plus, il existe chez les mammifères plusieurs protéines RFX qui sont requises pour l'assemblage des cils dans des territoires différents, ce qui peut aussi expliquer en partie le contrôle tissulaire de la ciliogenèse (Tableau l). D'autre part, FOXJl est indispensable à la différenciation des épithéliums multiciliés et à l'acquisition des propriétés des cils mobiles.

Plusieurs études suggèrent que ces familles de facteurs de transcription coopèrent dans la biogenèse des cils mobiles. $\varepsilon n$ effet, outre leur expression dans les tissus possédant des cils mobiles, les facteurs RFX régulent l'expression de gènes impliqués dans les fonctions des cils associées à leur motilité, tels que Dnah9 (dynein, axonemal heavy chain 9), qui est également une cible de FOXJ1 [27, 31]. Des expériences réalisées chez la souris, le poisson zèbre et dans les cellules respiratoires humaines, montrent que FOXJ1 induit l'expression des gènes RFX3 et RFX2 pendant la différenciation des cils mobiles $[24,26,32]$. Cependant, Rfx3 ne semble pas être régulé par FOXJ1 dans le plancher du tube neural, qui porte de longs monocils dont la fonction est encore débattue [33]. À l'inverse, dans les cellules épendymaires de souris, RFX3 se lie au promoteur du gène Foxj1 [12], suggérant l'existence d'une régulation croisée entre ces facteurs de transcription. II a également été montré que les facteurs RFX et FOXJl peuvent faire partie d'un même complexe transcriptionnel. Ainsi, dans les cellules respiratoires humaines, l'expression de FOXJ1 seule, et non celle de RFX3, est suffisante pour induire l'expression des gènes contrôlant la motilité ciliaire. Pourtant, l'expression des gènes cibles de FOXJI est augmentée en présence de RFX3 et les deux protéines humaines peuvent êtres coimmunoprécipitées lorsqu'elles sont surexprimées dans ces cellules [32]. Un crible double-hybride a également montré une interaction directe entre les protéines de souris RFX2 et FOXJ1 [34].

Les facteurs RFX et FOXJI peuvent également coopérer avec des facteurs de transcription spécifiques des tissus, et ainsi permettre une plus grande diversité ciliaire.

\section{Contrôle de la diversité ciliaire}

Spécificité ciliaire chez les invertébrés : coopération entre facteurs de transcription

De manière intéressante, l'utilisation des modèles invertébrés, qui possèdent un nombre restreint de cils 


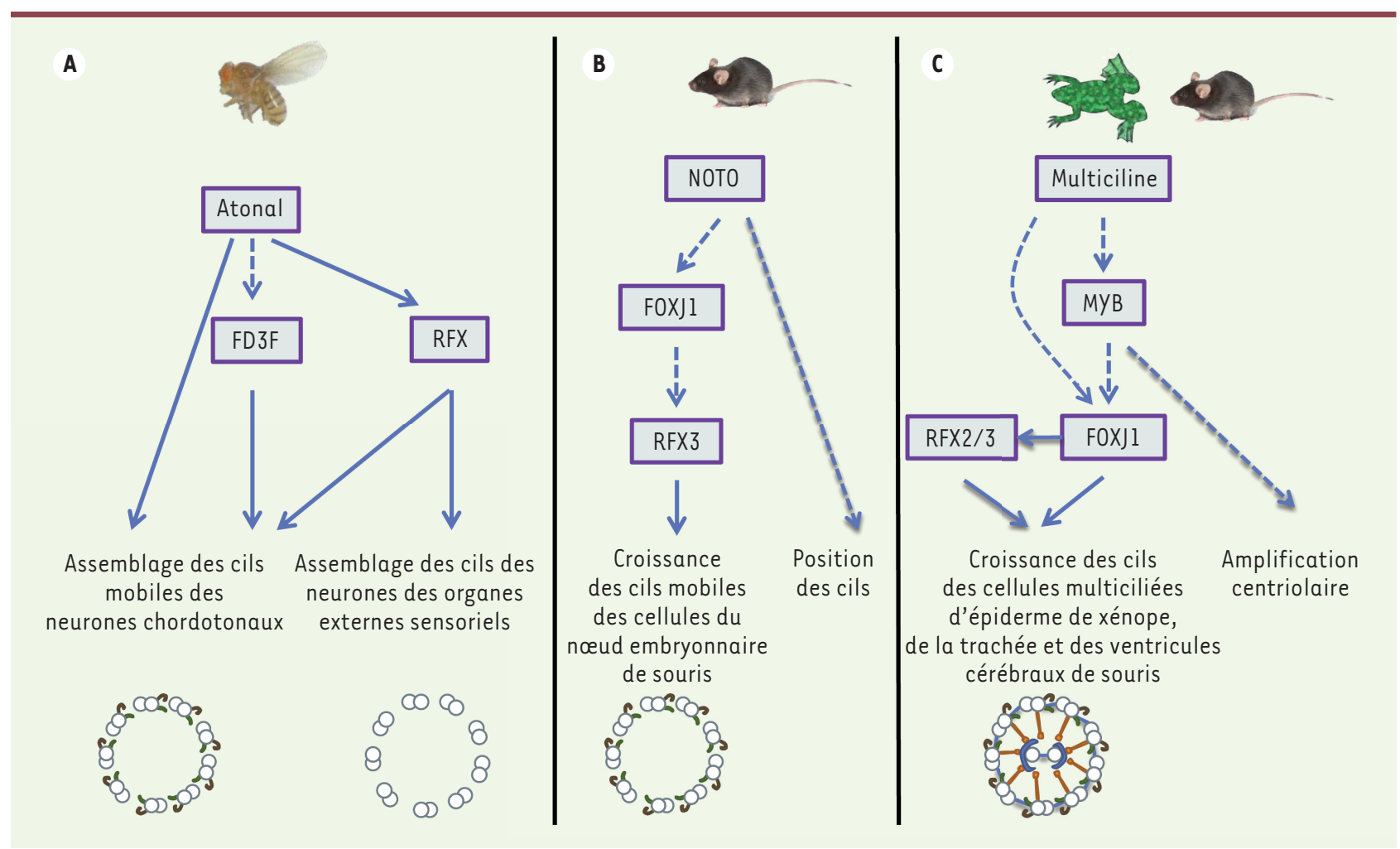

Figure 2. Exemples de régulation de l'expression des facteurs de transcription des familles RFX et FOXJ1. A. Chez la drosophile, la formation des deux types de cils sensoriels est différente. La fonction des cils des organes chordotonaux nécessite à la fois l'expression de $f d 3 f$ et de rfx, tous deux régulés par atonal. De plus, atonal peut réguler directement des gènes qui codent pour des protéines du cil. L'assemblage des cils des neurones des organes sensoriels externes ne requiert que l'expression de rfx. B. Dans les cellules du nœud embryonnaire de souris, lors de la mise en place du programme d'assemblage des cils, le facteur NOTO active Foxjl qui, en retour, régule positivement l'expression de Rfx3. NOTO régule la position des cils indépendamment de FOXJ1. C. L'expression de Foxjl, qui est nécessaire à la formation des multicils de l'épiderme de xénope et des épithéliums de la trachée et des ventricules cérébraux chez la souris, est sous le contrôle du facteur MYB, lui-même régulé par la protéine multiciline. La protéine MYB régule l'amplification centriolaire indépendamment de FOXJ1. RFX3 chez la souris et RfX2 chez le xénope sont requis, avec FOXJ1, pour réguler la croissance des cils de l'épithélium ventriculaire ou de l'épiderme, respectivement. Les flèches pleines traduisent des régulations directes. Les flèches pointillées des régulations indirectes ou encore à définir. Une représentation schématique de l'ultrastructure est rappelée pour chaque cil pris en exemple.

différents, a permis de mieux comprendre comment la spécificité ciliaire peut être contrôlée. Ainsi, les neurones chordotonaux chez la drosophile, qui servent à l'audition et à la proprioception, possèdent des cils mobiles en $(9+0)$ dont l'assemblage est contrôlé à la fois par les facteurs RFX et FD3F (forkhead domain 3F). Ces deux protéines coopèrent pour réguler des gènes spécifiques de ce type ciliaire, comme les gènes Nan, lav et Dhc93AB (dynein axonemal heavy chain $93 A B$ ), qui possèdent dans leurs séquences régulatrices des sites de liaison pour RFX et FD3F. Toute mutation dans le site de fixation de chacun de ces facteurs abolit ou diminue fortement l'expression de ces gènes cibles [23]. De plus, il a été montré que le niveau absolu de RFX dans les différents types de cellules ciliées de la drosophile avait une influence sur la nature des gènes induits. Le contrôle de l'expression de RFX par le facteur de transcription proneuronal atonal, spécifique des neurones chordotonaux, permet d'obtenir des niveaux élevés de RFX. Ces niveaux élevés sont en particulier nécessaires à l'assemblage de la racine ciliaire, qui ancre le cil dans le cytosquelette de la cellule et qui est très développée dans ce type cellulaire. Atonal peut directement activer l'expression du gène ciliaire dilatory, indépendamment du complexe transcriptionnel RFX/FD3F [35] et, ainsi, contrôler la spécialisation des cils des neurones chordotonaux (Figure 2A). Un mécanisme similaire semble opérer chez la souris au niveau des cils mobiles du nœud embryonnaire. En effet, leur formation nécessite le facteur de transcription NOTO (notochord homeobox). Celui-ci active directement FoxJl qui, en retour, active $R f x 3$ (Figure 2B). La réexpression de Foxjl chez un mutant Noto suffit à rétablir l'expression des gènes ciliaires, ainsi que la taille et le battement normal des cils $[24,26]$. Cependant, la polarisation du cil, c'est-à-dire sa localisation spécifique à la surface de la cellule, est altérée, indiquant que NOTO active, indépendamment de FOXJ1 et de RFX3, les gènes nécessaires au positionnement correct des cils. 


\section{Recrutement de facteurs de transcription accessoires}

La formation spécifique d'un type de cil ou d'un autre peut aussi faire appel à des facteurs de transcription accessoires, dont la plupart restent à identifier. Ainsi, il a été montré que les différents gènes requis pour l'assemblage de tous les cils chez $C$. elegans possèdent dans leur promoteur, en plus d'un motif de liaison des protéines RFX, un motif conservé appelé boîte $C$, qui est requis pour l'activation de ces gènes. Le (ou les) facteur qui pourrait contrôler l'expression des gènes ciliaires en utilisant ce motif reste néanmoins inconnu [36]. Toujours chez $C$. elegans, il a été montré que la protéine RFX (DAF-19) induit l'expression d'un autre facteur de transcription, FKH-2 (forkhead-2), spécifique d'un sous-type de cellule ciliée et qui lui confère son identité [37]. La raison pour laquelle ce facteur est induit uniquement dans un seul type de cil par DAF-19 reste inconnu, mais il est vraisemblable que des facteurs spécifiques de ce type cellulaire, encore à identifier, soient impliqués dans le processus. Chez la souris, quelques données suggèrent que de tels facteurs pourraient également participer aux processus de ciliogenèse. Par exemple, le régulateur transcriptionnel HNF1 $\beta$ (hepatocyte nuclear factor $1 \beta$ ), qui joue un rôle majeur dans l'homéostasie du rein, régule l'expression des gènes $P$ khdl (polycystic kidney and hepatic disease 1) et Pkd2 (polycystic kidney disease 2), indispensables à la détection du flux d'urine par le cil [38].

\section{Contrôle du nombre de cils}

Les mécanismes qui permettent de contrôler la formation d'un nombre important de cils mobiles à la surface d'une seule cellule d'un épithélium commencent à être bien compris chez le xénope et la souris. Deux protéines particulières, la multiciline et la protéine MYB (myeloblastosis protein), sont requises pour que les cellules développent des cils mobiles [27, 39]. La multiciline, une protéine nucléaire qui ne possède pas de domaine caractérisé de liaison à l'ADN, est nécessaire pour la différenciation des touffes de cils mobiles. Elle agit, entre autres, en induisant l'expression du facteur de transcription MYB qui, lui-même, est nécessaire à l'amplification centriolaire, étape préalable à la formation d'un grand nombre de cils. Ces protéines sont toutes deux impliquées dans la régulation de Foxjl (Figure 2C) qui permet, en coopération avec les facteurs RFX, d'induire la formation des cils mobiles. L'identification des mécanismes par lesquels la multiciline et MYB contrôlent l'expression de leurs gènes cibles devrait apporter des éléments importants pour comprendre la différenciation des épithéliums multiciliés. Ces résultats montrent que, si les facteurs RFX et FOXJI sont des effecteurs clés du programme de transcription qui gouverne la formation des cils, ils sont soumis eux-mêmes à un contrôle précis. Ce contrôle intègre par ailleurs plusieurs voies de signalisation majeures du développement comme les voies Notch, Wnt et FGF (fibroblast growth factor); ces voies ne seront pas détaillées ici (pour revue [1]).

\section{Identification des cibles des régulateurs ciliaires : de nouveaux gènes candidats de ciliopathies}

Des études génomiques et protéomiques visant à identifier les gènes cibles des facteurs de transcription ciliaires ont permis d'établir des listes exhaustives des composants des cils $([17,23,31,40,41]$ et pour revue $[1$,
42]). Ainsi, le gène ccdc151 (coiled-coil domain containing 151), identifié comme cible de RFX chez la drosophile [31], est nécessaire à la motilité des cils via le contrôle de l'assemblage des bras de dynéine chez le poisson zèbre [43]. II en est de même pour le gène chibby, également identifié comme cible de RFX, et qui joue un rôle crucial dans la biogenèse de la zone de transition chez la drosophile et pour la mise en place des cils mobiles chez la souris $[44,45]$. De la même manière, une étude visant à trouver les cibles de RFX2 dans les cellules multiciliées de la peau de xénope a conduit à l'identification de deux nouveaux gènes ciliaires. Le premier, ttc29 (tetratricopeptide repeat domain 29), est nécessaire à la régulation de l'IFT et le second, ribc2 (RIB43A domain with coiled-coils 2), est essentiel à la motilité des cils, et son absence perturbe l'organisation microtubulaire de l'axonème [17].

Ces études ont aussi permis d'identifier des gènes dont l'importance chez l'homme a été démontrée par leur implication dans plusieurs ciliopathies. Par exemple, le gène Zmynd10 (zinc finger, MYND-type containing 10), identifié comme cible des facteurs de transcription FD3F et RFX chez la drosophile, est essentiel à l'assemblage des bras de dynéine chez l'homme et la drosophile, et il est associé à des dyskinésies ciliaires primitives [46]. Ainsi, l'analyse des programmes transcriptionnels impliqués dans la ciliogenèse devrait permettre de mieux comprendre les mécanismes d'assemblage des cils et leur diversité au sein d'un organisme. La poursuite de la caractérisation des gènes cibles sous contrôle des différents programmes transcriptionnels devrait permettre d'aider à l'identification de nouveaux gènes impliqués dans les pathologies ciliaires chez l'homme. $\diamond$

\section{SUMMARY}

\section{Transcriptional control of ciliary genes}

Cilia are found in many eukaryotic species and share a common microtubule architecture that can nonetheless show very diverse features within one animal. The genesis of cilia and their diversity require the expression of different specific genes. At least two classes of transcription factors are involved in ciliogenesis: the RFX family, essential for the assembly of most cilia and the FOXJ1 transcription factors that are key regulators of motile cilia assembly. These two different families of transcription factors have both specific and common target genes and they can also cooperate for the formation of cilia. In collaboration with cell type specific factors, they also contribute to the specialisation of cilia. As a consequence, the identification of RFX and FOXJ1 target genes has emerged as an efficient strategy to identify novel ciliary genes, and in particular genes potentially implicated in ciliopathies. $\diamond$ 


\section{REMERCIEMENTS}

Nous remercions la Fondation pour la recherche médicale pour son soutien à Julie Jerber (prix Line Pomaret-Delalande) et à l'équipe dirigée par Bénédicte Durand (équipe FRM, DEQ20131029168). Jennifer Vieillard est financée par un contrat doctoral de l'université Lyonl.

\section{LIENS D'INTÉRÊT}

Les auteurs déclarent n'avoir aucun lien d'intérêt concernant les données publiées dans cet article.

\section{RÉFÉRENCES}

1. Choksi SP, Lauter G, Swoboda P, et al. Switching on cilia: transcriptional networks regulating ciliogenesis. Development $2014 ; 141$ : 1427-41.

2. Scholey JM. Intraflagellar transport motors in cilia: moving along the cell's antenna. J Cell Biol $2008 ; 180: 23-9$.

3. Satir P, Christensen ST. Overview of structure and function of mammalian cilia. Annu Rev Physiol $2007 ; 69: 377-400$.

4. Sulik K, Dehart DB, langaki T, et al. Morphogenesis of the murine node and notochordal plate. Dev Dyn $1994 ; 201: 260-78$.

5. Lidow MS, Menco BP. Observations on axonemes and membranes of olfactory and respiratory cilia in frogs and rats using tannic acid-supplemented fixation and photographic rotation.

J Ultrastruct Res $1984 ; 86: 18-30$.

6. Sobkowicz HM, Slapnick SM, August BK. The kinocilium of auditory hair cells and evidence for its morphogenetic role during the regeneration of stereocilia and cuticular plates. J Neurocytol 1995 ; $24: 633-53$.

7. Chu JSC, Baillie DL, Chen N. Convergent evolution of RFX transcription factors and ciliary genes predated the origin of metazoans. BMC Evol Biol $2010 ; 10: 130$.

8. Swoboda P, Adler HT, Thomas JH. The RFX-type transcription factor DAF-19 regulates sensory neuron cilium formation in C. elegans. Mol Cell $2000 ; 5: 411-21$.

9. Dubruille R, Laurençon A, Vandaele $C$, et al. Drosophila regulatory factor $X$ is necessary for ciliated sensory neuron differentiation. Development $2002 ; 129$ : 5487-98.

10. Bonnafe $\varepsilon$, Touka M, AitLounis A, et al. The transcription factor RFX3 directs nodal cilium development and left-right asymmetry specification. Mol Cell Biol $2004 ; 24$ : 4417-27.

11. Ait-Lounis A, Baas D, Barras $\varepsilon$, et al. Novel function of the ciliogenic transcription factor RFX3 in development of the endocrine pancreas. Diabetes $2007 ; 56: 950-9$.

12. Zein $L$ El, Ait-Lounis A, Morlé L, et al. RFX3 governs growth and beating efficiency of motile cilia in mouse and controls the expression of genes involved in human ciliopathies. J Cell Sci 2009; 122 : 3180-9.

13. Baas D, Meiniel A, Benadiba C, et al. A deficiency in RFX3 causes hydrocephalus associated with abnormal differentiation of ependymal cells. Eur J Neurosci $2006 ; 24: 1020-30$.

14. Ashique AM, Choe $\mathrm{Y}$, Karlen M, et al. The Rf $x 4$ transcription factor modulates Shh signaling by regional control of ciliogenesis. Sci Signal $2009 ; 2$ : ra70.

15. Bisgrove BW, Makova S, Yost HJ, et al. RFX2 is essential in the ciliated organ of asymmetry and an RFX2 transgene identifies a population of ciliated cells sufficient for fluid flow. Dev Biol 2012 ; 363: 166-78.

16. Chung MI, Peyrot SM, LeBoeuf $S$, et al. RFX2 is broadly required for ciliogenesis during vertebrate development. Dev Biol $2012 ; 363: 155-65$

17. Chung MI, Kwon T, Tu F, et al. Coordinated genomic control of ciliogenesis and cell movement by RFX2. Elife $2014 ; 3$ : e01439.

18. Manojlovic Z, Earwood R, Kato A, et al. RFX7 is required for the formation of cilia in the neural tube. Mech Dev 2014 ; 132 : 28-37.

19. Jerber J, Thomas J, Durand B. Contrôle transcriptionnel de la ciliogenèse au cours du développement animal. Biol Aujourdhui 2012 ; 206 : 205-18.

20. Blackshear PJ, Graves JP, Stumpo DJ, et al. Graded phenotypic response to partial and complete deficiency of a brain-specific transcript variant of the winged helix transcription factor RFX4. Development $2003 ; 130: 4539-52$.

21. Kistler WS, Horvath GC, Dasgupta A, et al. Differential expression of Rfxl-4 during mouse spermatogenesis. Gene Expr Patterns 2009 ; 9 : 515-9.

22. Vij S, Rink JC, Ho HK, et al. Evolutionarily ancient association of the FoxJl transcription factor with the motile ciliogenic program. PLoS Genet $2012 ; 8$ : el003019.

23. Newton FG, zur Lage PI, Karak S, et al. Forkhead transcription factor Fd3F cooperates with Rfx to regulate a gene expression program for mechanosensory cilia specialization. Dev Cell $2012 ; 22$ : 1221-33.

24. Alten L, Schuster-Gossler K, Beckers A, et al. Differential regulation of node formation, nodal ciliogenesis and cilia positioning by Noto and Foxj1. Development $2012 ; 139: 1276-84$.

25. Brody SL, Yan XH, Wuerffel MK, et al. Ciliogenesis and left-right axis defects in forkhead factor HFH-4-null mice. Am J Respir Cell Mol Biol $2000 ; 23: 45-51$.

26. $\mathrm{Yu}_{\mathrm{u}} \mathrm{X}, \mathrm{Ng} \mathrm{CP}, \mathrm{Habacher} \mathrm{H}$, et al. Foxjl transcription factors are master regulators of the motile ciliogenic program. Nat Genet $2008 ; 40: 1445-53$.
27. Stubbs JL, Oishi I, Izpisúa Belmonte JC, et al. The forkhead protein Foxj1 specifies node-like cilia in Xenopus and zebrafish embryos. Nat Genet 2008 ; $40: 1454-60$

28. Hagenlocher C, Walentek P, M Ller C, et al. Ciliogenesis and cerebrospinal fluid flow in the developing Xenopus brain are regulated by foxj1. Cilia $2013 ; 2: 12$.

29. Horani A, Ferkol TW, Shoseyov D, et al. LRRC6 mutation causes primary ciliary dyskinesia with dynein arm defects. PLoS One 2013 ; 8 : e59436.

30. You Y, Huang $T$, Richer $\varepsilon$, et al. Role of $f$-box factor foxjl in differentiation of ciliated airway epithelial cells. Am J Physiol Lung Cell Mol Physiol 2004 ; $286:$ L650-7.

31. Laurençon A, Dubruille R, Efimenko $\varepsilon$, et al. Identification of novel regulatory factor $X(R F X)$ target genes by comparative genomics in Drosophila species. Genome Biol 2007 ; 8 : R195.

32. Didon L, Zwick RK, Chao IW, et al. RFX3 modulation of FOXJ1 regulation of cilia genes in the human airway epithelium. Respir Res $2013 ; 14: 70$.

33. Cruz C, Ribes V, Kutejova $\varepsilon$, et al. Foxjl regulates floor plate cilia architecture and modifies the response of cells to sonic hedgehog signalling. Development $2010 ; 137: 4271-82$.

34. Ravasi T, Suzuki H, Cannistraci CV, et al. An atlas of combinatorial transcriptional regulation in mouse and man. Cell 2010 ; 140 : 744-52.

35. Cachero S, Simpson TI, Lage PI Zur, et al. The gene regulatory cascade linking proneural specification with differentiation in Drosophila sensory neurons. PLoS Biol 2011 ; 9 : el 1000568.

36. Burghoorn J, Piasecki BP, Crona F, et al. The in vivo dissection of direct RFXtarget gene promoters in $C$. elegans reveals a novel cis-regulatory element, the C-box. Dev Biol $2012 ; 368: 415-26$.

37. Mukhopadhyay S, Lu Y, Qin H, et al. Distinct IFT mechanisms contribute to the generation of ciliary structural diversity in C. elegans. EMBO J $2007 ; 26$ : 2966-80.

38. Gresh L, Fischer $\varepsilon$, Reimann A, et al. A transcriptional network in polycystic kidney disease. EMBO J $2004 ; 23: 1657-68$.

39. Tan FE, Vladar $\varepsilon K, M a ~ L$, et al. Myb promotes centriole amplification and later steps of the multiciliogenesis program. Development 2013; 140 : 4277-86.

40. Chen N, Mah A, Blacque $0 \varepsilon$, et al. Identification of ciliary and ciliopathy genes in Caenorhabditis elegans through comparative genomics. Genome Biol $2006 ; 7$ : R126.

41. Efimenko $\varepsilon$, Bubb K, Mak Hy, et al. Analysis of xbx genes in C. elegans. Development $2005 ; 132$ : 1923-34.

42. Thomas J, Morlé L, Soulavie F, et al. Transcriptional control of genes involved in ciliogenesis: a first step in making cilia. Biol Cell $2010 ; 102: 499-513$.

43. Jerber J, Baas D, Soulavie F, et al. The coiled-coil domain containing protein CCDCl51 is required for the function of IFT-dependent motile cilia in animals. Hum Mol Genet $2014 ; 23: 563-77$.

44. Enjolras C, Thomas J, Chhin B, et al. Drosophila chibby is required for basal body formation and ciliogenesis but not for Wg signaling. J Cell Biol 2012 ; $197: 313-25$.

45. Voronina V, Takemaru KI, Treuting P, et al. Inactivation of Chibby affects function of motile airway cilia. J Cell Biol $2009 ; 185: 225-33$.

46. Moore DJ, Onoufriadis A, Shoemark A, et al. Mutations in ZMYND10, a gene essential for proper axonemal assembly of inner and outer dynein arms in humans and flies, cause primary ciliary dyskinesia. Am J Hum Genet 2013; $93: 346-56$.

47. Liu Y, Pathak N, Kramer-Zucker A, et al. Notch signaling controls the differentiation of transporting epithelia and multiciliated cells in the zebrafish pronephros. Development 2007 ; 134 : 1111-22.

48. Fort $C$, Bastin $P$. Élongation de l'axonème et dynamique du transport intraflagellaire. Med Sci (Paris) 2014 ; 30 : 955-61.

49. Diguet N, Meilhac SM. Cils et morphogenèse cardiaque. Med Sci (Paris) $2014 ; 30$ : 996-1003.

50. Ezan J, Montcouquiol M. Les liens multiples entre les cils et la polarité planaire cellulaire. Med Sci (Paris) 2014 ; 30 : 1004-10.

51. Benmerah $\mathrm{A}$. La poche ciliaire : fruit des liaisons du centrosome avec le trafic vésiculaire. Med Sci (Paris) 2014 ; 30 : 962-7. 